\title{
Crecimiento económico y desarrollo humano en la ciudad de México con respecto a un entorno nacional: una perspectiva neoclásica y dualista
}

\section{Economic growth and human development in Mexico city with respect to the national sphere: a neoclassical and dualist perspective}

\begin{abstract}
Gerardo Ángeles-Castro*
Abstract

Mexico city is no longer the dominant pole that boosted social and economic polarization. According to the dualist thesis, over the longer run a dominant pole can exert a spread effect. The Federal District keeps the highest development indicators, but it has the lowest growth in Mexico. Although neoclassical growth theory can justify this trend, international comparisons show that the economic and human development indicators in Mexico city have fallen behind; moreover the parametric analysis does not find evidence that the city has exerted a spread effect on the national territory in the last decades.
\end{abstract}

Keywords: dualism, Mexico city, neoclassical theory, economic growth, human development.

\section{Resumen}

La ciudad de México ya no es el polo dominante que propicia polarización socioeconómica, según la teoría dualista, un polo concentrador; en el largo plazo puede convertirse en un polo diseminador. Ciertamente mantiene los indicadores de desarrollo más altos, pero éstos tienen el crecimiento más lento a nivel nacional. Aunque la teoría neoclásica justifica esta tendencia, las comparaciones internacionales muestran que tiene rezagos en la expansión de sus indicadores, a su vez, el análisis paramétrico no encuentra evidencia de que la ciudad ejerza, en décadas recientes, un efecto diseminador a nivel nacional.

Palabras clave: dualismo, ciudad de México, teoría neoclásica, crecimiento económico, desarrollo humano. 


\section{Introducción}

Después de haber alcanzado la mayor participación en el Producto Interno Bruto (PIB) nacional en 1960, la ciudad de México tiende a disminuir su influencia en la actividad económica del país, la reducción de la concentración económica y de bienestar se evidencia con lentas tasas de crecimiento económico y desarrollo humano: las más bajas a nivel nacional.

De acuerdo con las ideas dualistas, un polo concentrador en el largo plazo puede convertirse en un polo diseminador de crecimiento y desarrollo por tendencia natural y por la acción de políticas públicas de desconcentración y regionalización. Sin embargo, para que una región pueda ejercer un efecto catalizador debe mantener un crecimiento, de tal forma que no regrese a ser un polo concentrador; a la vez, el crecimiento tiene que ser lo suficientemente robusto para diseminar desarrollo en el resto de las regiones.

Dado que la expansión de indicadores de crecimiento y desarrollo en la ciudad de México ha sido la más lenta a nivel nacional en los años que abarca el análisis, es importante evaluar si su expansión es tan sólida como para garantizar que la capital pueda ejercer el papel de polo catalizador a nivel nacional. Lo anterior se puede justificar mediante postulados neoclásicos, en el sentido de que éstos argumentan que los países o regiones con un mayor nivel de desarrollo inicial tienden a crecer más lento, en relación con los que tienen un nivel de desarrollo inicial más bajo.

El objetivo de esta investigación es evaluar si el lento crecimiento de la ciudad de México está asociado con un proceso de convergencia neoclásico, y si éste puede ser justificado mediante un análisis comparativo de evidencia empírica internacional. Adicionalmente se evalúa si el crecimiento de la ciudad es lo suficientemente robusto para ejercer un proceso de diseminación dualista, mediante el cual se impulse el desarrollo nacional.

El principal argumento es que la capital de México tiende a abandonar el papel de polo concentrador y además crece de manera muy lenta en los últimos años. De tal forma que no tiene posibilidades de ejercer un efecto diseminador en el resto del país, por lo que no propicia la convergencia a nivel nacional.

Es importante recalcar que el efecto diseminador no significa crear mayor divergencia entre la capital y el resto de las regiones, por el contrario, implica catalizar el desarrollo en el interior del país, aunque para ello la ciudad de México necesita mantener índices de crecimiento y desarrollo robustos. Asimismo, para evitar o disminuir la polarización es necesario impulsar políticas de descentralización y desarrollo equitativo entre las regiones. 
La presente investigación se estructura de la siguiente forma: en la segunda sección se realiza una comparación entre los argumentos dualistas y el desarrollo histórico en la ciudad de México, se comenta brevemente sobre los postulados neoclásicos en términos de diferencias en tasas de crecimiento; la tercera parte ilustra la evolución de indicadores demográficos y educativos en dicha ciudad y su comparación a nivel nacional; el cuarto punto incluye indicadores económicos en tal demarcación y su comparación con el resto de la república; la quinta presenta la evolución del índice de desarrollo humano (IDH) en la capital en comparación con el ámbito nacional; adicionalmente muestra el IDH por delegación y se compara con otras naciones; la sexta sección contrasta los de indicadores económicos y del IDH de la ciudad de México con el resto de las entidades federativas y a nivel internacional; la séptima parte incorpora un análisis econométrico para analizar la causalidad de crecimiento económico y la convergencia del pIB per cápita entre la ciudad de México y el resto de las entidades federativas. Finalmente, se integran las conclusiones de la investigación.

\section{Dualismo y neoclasicismo}

El término dualismo describe una situación del proceso de desarrollo en la cual pueden darse diferencias sociales, económicas y tecnológicas entre regiones de un mismo país. Las discrepancias comúnmente se presentan entre el sector rural y el urbano o industrializado, y se aprecian, principalmente, por la brecha de los ingresos per cápita y el crecimiento económico entre regiones (Thirlwall, 2006). Las causas fundamentales del dualismo son las diferencias culturales, costumbres y actitudes entre sistemas sociales nativos y sistemas sociales impuestos o importados. Cada uno de estos sistemas sociales se acompañan de procesos de especialización diferentes, en particular los impuestos se auxilian de tecnologías del exterior y tienen formas de especialización más sofisticadas, lo cual acentúa la brecha económica y tecnológica. Adicionalmente el dualismo se presenta por una tendencia a concentrar geográficamente el desarrollo tecnológico, económico, social y humano en las regiones favorecidas, dando como resultado mayor divergencia entre zonas. En este sentido, y desde un punto de vista más extremo, en el dualismo se privilegia el desarrollo de ciertos polos y se margina a las zonas geográficas que no se consideran prioritarias. Asimismo, que la población nativa se resista a formas de desarrollo impuestas, con tal de no perder su espacio cultural y no caer en sometimientos, es una forma de propiciar diferencias. El dualismo también puede emerger de manera natural debido a diferentes niveles de desarrollo geográfico o diferentes formas de especialización. 
Una vez que el proceso de divergencia o polarización se encuentra en marcha se puede acrecentar mediante flujos migratorios, es decir, si los procesos de producción en la región rural aplican técnicas con intensidad de mano de obra y en la región urbana o industrializada emplean mano de obra mejor remunerada, se crean incentivos para emigrar a la región con mayores posibilidades de ingreso; a su vez, si el polo concentrador presenta índices más altos de ingreso per cápita, crecimiento económico e industrialización, y tiende a concentrar el comercio, la inversión, el empleo y el desarrollo humano, entonces se generan más estímulos para la migración del campo a la ciudad. Es importante resaltar que además de la migración laboral se presentan movimientos de capital y de actividad empresarial; esta tendencia reduce en términos relativos la demanda, la inversión, el comercio y el empleo en las zonas rezagadas y crea mayor concentración y expansión en las zonas favorecidas. El impacto de la migración hacia las regiones en expansión también induce mejoras en el transporte, comunicaciones, educación, salud y, en general, en la calidad de vida. Gunnar Myrdal (1957) llama causalidad acumulativa al efecto que favorece a las regiones concentradoras y argumenta que este proceso puede ocasionar rezago en las otras regiones y, por lo tanto, hace que las diferencias de desarrollo persistan e incluso crezcan.

Albert O. Hirschman (1958) llama efecto polarizador al proceso que crea divergencia entre regiones y efecto diseminador a las repercusiones favorables que emanan de las regiones en expansión. El segundo consiste, esencialmente, en un incremento en la demanda de productos de las áreas en rezago y de la difusión de tecnología y conocimiento. Myrdal (1957) argumenta que el efecto diseminador es más débil que el efecto polarizador y añade que para reducir las diferencias interregionales se requiere la intervención del Estado a través de políticas regionales. Alternativamente se puede optar por esperar a que el efecto polarizador y el proceso de causalidad acumulativa se terminen en el largo plazo de manera natural, lo cual puede ser una transición lenta.

El proceso natural, eventualmente, se presenta cuando los costos relativos en la región en expansión se incrementan, principalmente por el congestionamiento económico y social, al grado de que se desacelera el crecimiento. Adicionalmente la inmigración se frena e incluso se revierte. Sin embargo, la expansión en la región favorecida debe continuar, aunque en menor escala, de manera tal que el efecto diseminador se refuerce y a su vez propicie convergencia entre regiones (Saldaña, 2008), de esta forma, la región favorecida deja de operar como polo concentrador y se convierte en un polo catalizador que mantiene una demanda sólida por los productos de las otras regiones, además, mantiene la difusión de tecnología, inversiones y desarrollo humano (Ranis, 2003). 
La evolución socioeconómica de la ciudad de México presenta semejanzas con el concepto de dualismo. Durante la época colonial la polarización regional fue evidente, la capital de la Nueva España, en la cual se impuso un sistema de desarrollo ajeno, concentró el poder sociopolítico y económico; aunque este sistema importado se extendió a otras regiones se concentró de manera notoria en la capital; diversas regiones fueron relegadas mientras que varias culturas nativas permanecieron al margen para evitar ser sojuzgadas o exterminadas por el poder colonial; incluso, el basto territorio del norte de la Nueva España permaneció deshabitado y desconectado del poder central; de esta manera, el desarrollo de una región se daba a expensas o al margen de otras, lo que fomentó la polarización regional.

$\mathrm{Al}$ inicio de la época del México independiente las formas de organización regional no cambiaron significativamente, la inversión, la economía, la política, los servicios educativos y de salud se aglomeraban principalmente en la capital de la naciente república, esto propició un fuerte flujo migratorio hacia la ciudad de México y por consiguiente, el proceso de causalidad acumulativa y el efecto polarización se reafirmaron. Los programas regionales y de desconcentración que se aplicaron a lo largo de los diferentes gobiernos del México independiente fueron endebles y arrojaron mínimos resultados.

En 1940 la ciudad de México participaba con 33.5\% del piв nacional, para 1960 esta participación alcanzó su mayor nivel llegando a 37.3\%, lo cual evidenciaba la concentración. La polarización y divergencia también eran evidentes, entre 1951 y 1960 el crecimiento promedio anual en la ciudad de México era de $8.06 \%$ mientras que en el resto del país era de 5.16\% (Germán, 2005). Después de 1960, la concentración económica empezó a ceder y la brecha del pIB entre la capital y el promedio del resto de las regiones disminuyó. Por ejemplo, entre 1971 y 1980, el PIB promedio anual en la ciudad de México crecía $5.8 \%$ y en las demás localidades crecía $7.0 \%$, una diferencia de 1.2 puntos porcentuales. Entre 1991 y 2000 el crecimiento de la ciudad de México permanecía menor con respecto al resto de las localidades aunque la diferencia era mínima. Sin embargo entre 2000 y 2006 el рIв promedio anual en la capital del país crecía $1.7 \%$, mientras que en el resto de la república la cifra era de $3.2 \%$, es decir, la diferencia se incrementaba a 1.5 puntos porcentuales, mientras que la participación de la ciudad de México en el PIB nacional caía a 20.3 por ciento. Bajo estas circunstancias es importante analizar si la ciudad de México logra mantener una expansión lo suficientemente sólida en los últimos años, para consolidar su efecto diseminador y ejercer el rol de polo catalizador. Para este fin, en las siguientes secciones se analiza la evolución de indicadores demográficos, de ingreso y de componentes de desarrollo humano de manera comparativa. 
Por otra parte, los postulados neoclásicos argumentan que los países o las regiones con un bajo nivel de ingreso per cápita inicial tienden a crecer más aceleradamente, en el largo plazo, que los países o regiones con un nivel más alto de ingreso per cápita inicial. Este proceso genera convergencia del ingreso; lo anterior se explica porque en la teoría neoclásica se asume que el capital tiene rendimientos decrecientes, es decir, el capital en un país con abundancia de esta variable generará menor cantidad de producto que el capital en un país con escasez del mismo; dado que en una función de producción neoclásica el factor capital tiene un papel relevante, entonces sus rendimientos afectarán de manera directa el crecimiento de un país o una región (Solow, 1956; Swan, 1956; Barro y Sala-i-Martin, 2003).

Si al capital le asignamos una composición más amplia y consideramos que además de tener un componente económico también tiene un componente humano, entonces el desarrollo humano -el cual se integra con los factores ingreso, salud y educación- debe crecer más lento en países o regiones con un nivel inicial alto de desarrollo.

$\mathrm{Al}$ igual que los conceptos dualistas, los postulados neoclásicos encuentran sustento empírico en la evolución de indicadores en la ciudad de México. Históricamente, la capital ha tenido los más altos niveles de ingreso per cápita, de escolaridad y en general de desarrollo, asimismo, en los últimos años, la expansión económica y del IDH en la ciudad de México ha sido la más lenta del país. En las siguientes secciones se muestra la evolución de indicadores en la capital mexicana, y se analiza si su lento crecimiento se puede justificar mediante los postulados neoclásicos o si tienen potencial para crecer más aceleradamente.

\section{2. Índices demográficos y educativos}

Entre 2000 y 2007 la población a nivel nacional aumentó 7.47\%, mientras que en la ciudad de México la cifra fue de sólo 1.49 por ciento. En general la tasa de crecimiento poblacional se desacelera pero en la capital nacional ha caído a niveles muy bajos; entre 2001 y 2007 esta tasa pasó de 1.33 a 0.86 a nivel nacional, mientras que en la ciudad de México fue de 0.44 a 0.08 en el mismo periodo. La gráfica I muestra la evolución de estas tasas.

Esta tendencia se explica porque entre 2000 y 2007 la tasa de mortalidad en el país se incrementó de 4.67 a 4.89 y en la capital nacional el aumento fue mayor: de 4.91 a 5.47 , mientras que la tasa de natalidad cayó de 23.75 a 18.60 en el país, y en la capital la cifra disminuyó de 18.45 hasta 14.80 . 


\section{Gráfica I \\ Tasas de crecimiento poblacional}

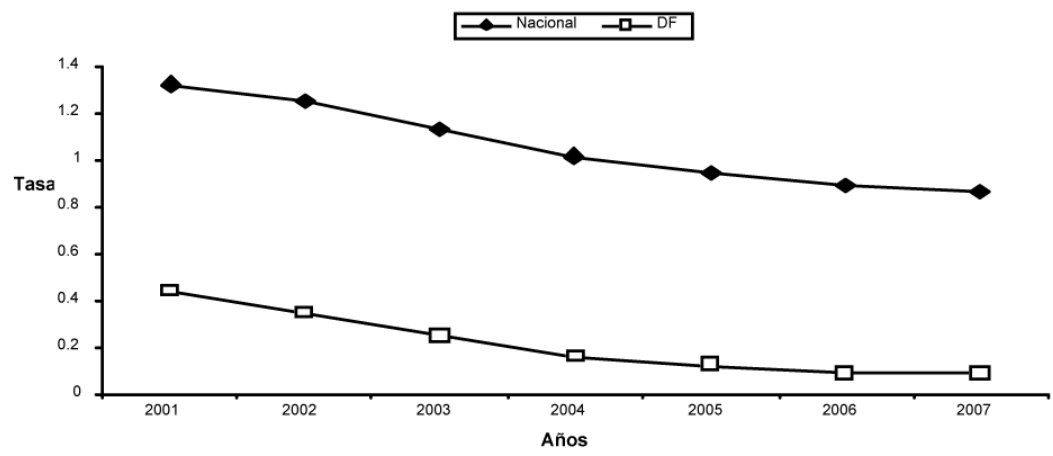

Fuente: De 2001 a 2004: Presidencia de la república, Sexto Informe de Gobierno del Presidente de los Estados Unidos Mexicanos, (2006); 4-15, de 2005 a 2007: Conapo, Proyecciones de la Población de México 2005-2050, (2008).

Por otra parte, la población en la ciudad de México envejece más rápido que en la media nacional. Entre 2000 y 2006 la población de 0 a 14 años se redujo $4.15 \%$ a nivel nacional y en la capital la caída fue mayor: 9.98\%, mientras que la población de adultos mayores, 65 años o más, creció de manera similar. Es importante mencionar que la fuerza laboral potencial, es decir la población entre 15 y 64 años, aumentó más lento en la capital nacional, en el mismo periodo incrementó sólo 4.2\%, mientras que en la media nacional el aumento fue de $11.31 \%$ (Presidencia de la república, 2006: 4-15; Conapo, 2008). En general, las cifras de la evolución de la población y su composición demuestran que la ciudad de México pierde su bono poblacional de manera más acelerada que la media nacional, lo cual tiende a disminuir sus posibilidades de crecimiento en términos relativos.

Previamente se ha mencionado que la población de 0 a 14 años ha decrecido tanto en el Distrito Federal como en la media nacional, pese a esta caída en este segmento poblacional la matrícula en educación básica a nivel nacional, en el periodo 2000-2006, creció en 6.90\%, por el contrario, en la ciudad de México cayó $1.05 \%$, estas tendencias se observan en la gráfica II. Aunque esto, en parte, se explica por la correlación entre el segmento de la población de cero a 14 años y la matrícula en educación básica.

En lo que respecta a la matrícula en educación media superior, en el país se presentó un notorio incremento de $27.66 \%$, es decir, mayor que el crecimiento del segmento poblacional de 15 a 64 años; mientras que en la ciudad de México el aumento fue de sólo 3.61\%, lo cual es menor 


\section{Gráfica II \\ Matrícula en educación básica (miles de alumnos)}

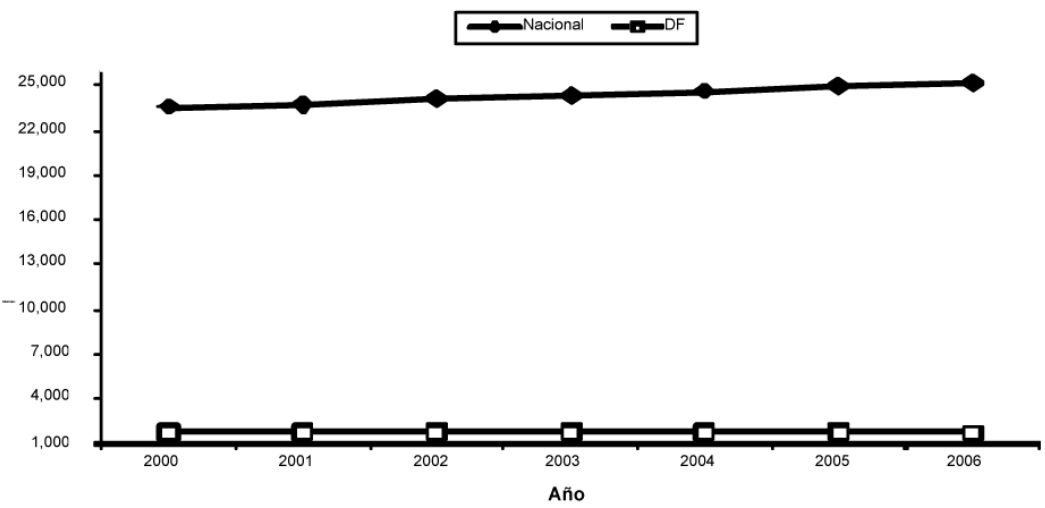

Fuente: Secretaría de Educación Pública, Estadísticas Históricas por estado, 2008.

\section{Gráfica III \\ Matrícula en educación media superior (miles de alumnos)}

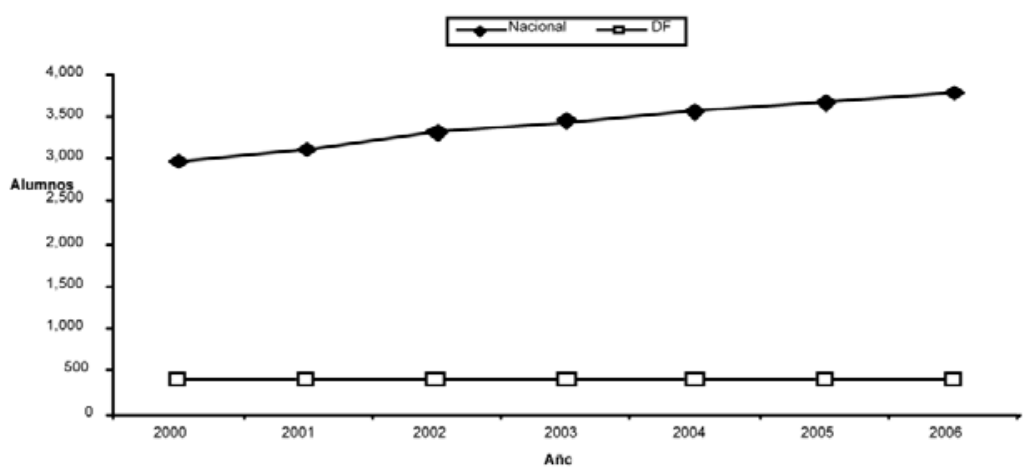

Fuente: Secretaría de Educación Pública, Estadísticas Históricas por Estado, 2008.

que el aumento del segmento poblacional de 15 a 64 años. En la gráfica III se observa la evolución de la matrícula en educación media superior.

La gráfica vi ilustra que la matrícula en educación superior también muestra un alto crecimiento a nivel nacional: $23.05 \%$, y un crecimiento modesto en la ciudad de México: 3.63\%. Las cifras de matrícula en educación media superior y superior revelan que en la ciudad de México no se logra incrementar los niveles educativos entre la población con la velocidad con que se hace en la media nacional.

Los efectos de la tendencia de la matrícula en los diferentes niveles educativos se ven reflejados en la escolaridad promedio. La media nacio- 


\section{Gráfica IV \\ Matrícula en educación superior \\ (miles de alumnos)}

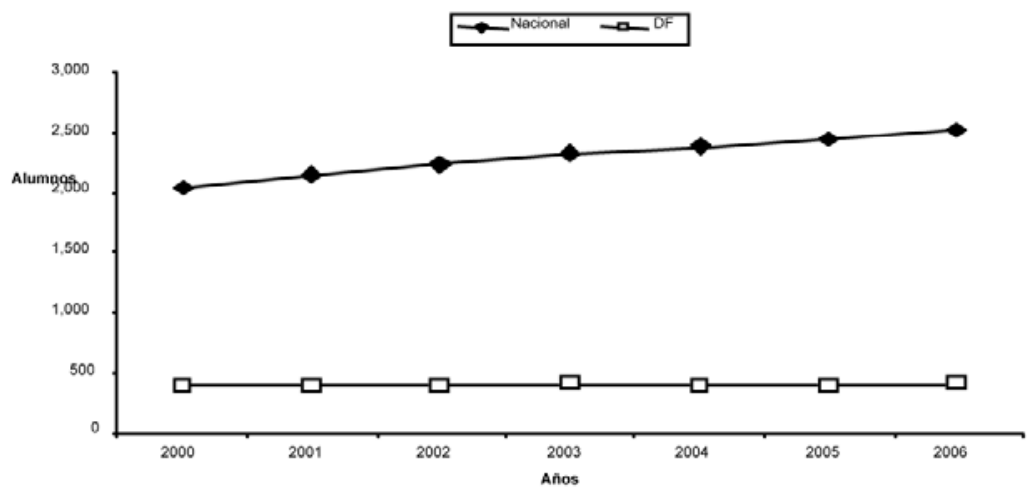

Fuente: Secretaría de Educación Pública, Estadísticas Históricas por estado, 2008.

nal se incrementó de 7.45 a 8.27 años, es decir, creció 0.82 años, mientras que la escolaridad promedio en la ciudad de México aumentó en menor proporción, 0.65 años, de 9.61 a 10.26 (Secretaría de Educación Pública, 2008). La capital mantiene el nivel más alto de escolaridad promedio, pero su crecimiento es lento, por lo cual tiende a rezagarse.

\section{Indicadores económicos}

En lo que respecta a indicadores económicos, la ciudad de México muestra un desempeño menos favorable que el resto de las entidades federativas: entre el año 2000 y 2006 el PIB creció en $1.74 \%$, promedio anual, y en el resto de las entidades aumentó 3.25\%. La evolución del PIB se muestra en la gráfica $\mathrm{v}$.

La capital mantiene el PIB per cápita más alto de la república. En 2006 éste fue de 38,976 pesos anual a precios de 1993, y en el país, excluyendo al Distrito Federal, fue de 13,652 pesos, una diferencia de casi tres a uno. Sin embargo, es importante notar que el ingreso per cápita en la ciudad de México creció $0.81 \%$, promedio anual en la capital, entre 2000 y 2006, y en el resto del país la cifra fue de 1.48 por ciento. La gráfica VI muestra la evolución del PIB per cápita.

Este lento crecimiento en la capital nacional se refleja en mayores rezagos en cuanto a creación de empleos. La tasa general de desempleo abierto en la ciudad de México es mayor y crece más rápido. Entre 2000 y 2004 este indicador pasó de 3.54 a 5.93 en dicha demarcación, es decir, incrementó 2.39 puntos, mientras que el promedio nacional pasó 


\section{Gráfica V PIB \\ (millones, precios de 1993)}

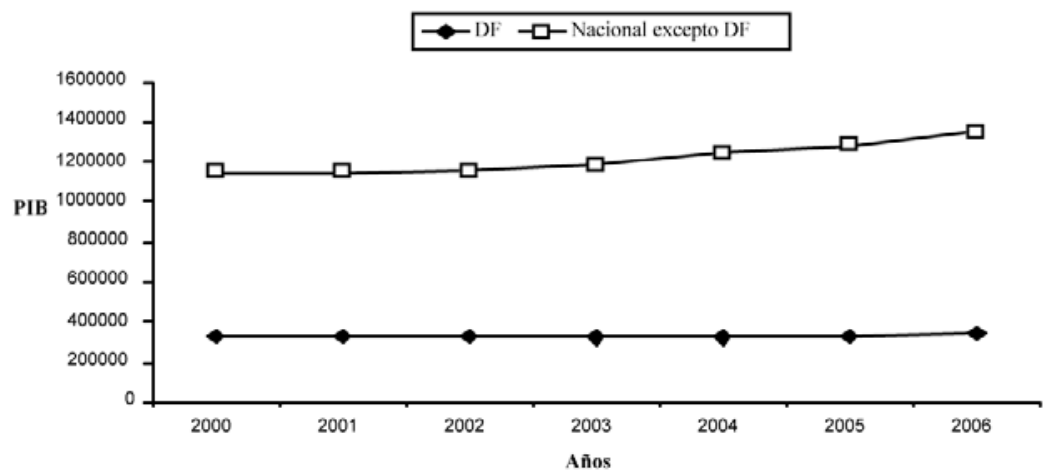

Fuente: Instituto Nacional de Estadística, Geografía e Informática (INEGI), Banco de Información Económica, 2008.

\section{Gráfica VI \\ PIB per capita \\ (precios 1993)}

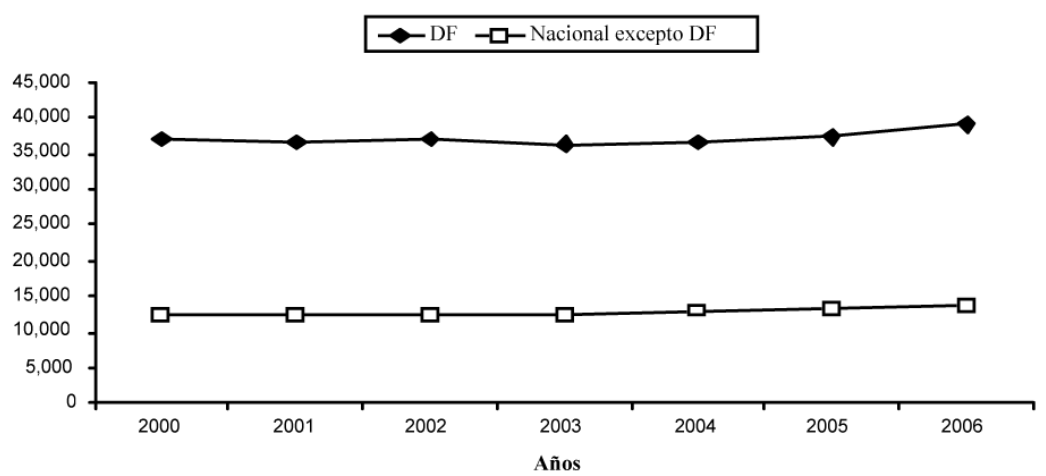

Fuente: Instituto Nacional de Estadística, Geografía e Informática (INEGI), Banco de Información Económica, 2008.

de 2.2 a $3.77 \%$, lo cual representa un crecimiento menor: 1.57 puntos, como se aprecia en la gráfica VII (INEGI, 2008).

\section{Desarrollo humano}

Existen diferentes índices para medir el desarrollo humano y la calidad de vida. Al respecto, el Consejo Nacional de Población (2008a) publica el índice de marginación estatal y municipal, el cual incluye indicadores como nivel de educación, analfabetismo, dotación de servicios públicos, 


\section{Gráfica VII \\ Tasa general de desempleo abierto}

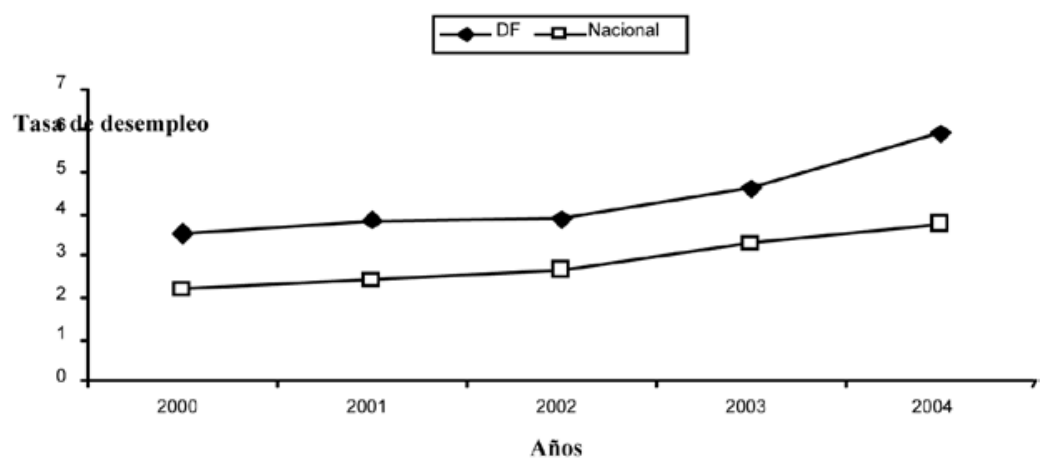

Fuente: United Nations Development Programme, Human Development Report-Mexico 2006-2007Migration and Human Development, 2007.

pobreza y calidad de la vivienda. Aunque el índice de marginación se calcula con una metodología sólida no se publica a nivel internacional por lo que no permite comparaciones de México con otros países. El Índice de Desarrollo Humano, construido por United Nations Development Programme (UNDP), se publica a nivel países, estados, municipios y regiones de manera periódica, e incluye tres principales componentes: salud, educación e ingreso y aunque no considera otros indicadores como seguridad o servicios públicos es, de cualquier forma, un índice respaldado por una sólida metodología, con un amplio número de observaciones en diferentes niveles, lo que permite hacer comparaciones nacionales e internacionales y valorar su evolución a través del tiempo. Dadas las ventajas del IDH, en este apartado se utiliza como base de datos para la elaboración de los diferentes análisis.

Entre 2000 y 2004 el IDH crece tanto a nivel nacional como en la ciudad de México, sin embargo, esta demarcación tiene el crecimiento más lento de todas las entidades del país. En los rubros de salud y educación del IDH, la ciudad de México también tiene el crecimiento más lento y en el componente de ingreso la variación es incluso negativa (UNDP, 2007: 174-175). El cuadro 1 muestra el IDH y sus componentes en los años de 2000 y 2004 y la variación porcentual tanto para la ciudad de México como para la media nacional.

El IDH en la ciudad de México, no sólo tiene el crecimiento más lento del país sino que también se hace más desigual. De acuerdo con el reporte Índice de Desarrollo Humano municipal en México (UndP, 2008), 13 delegaciones políticas del Distrito Federal cayeron en el lugar que ocupan en la clasificación nacional entre 2000 y 2005, y muchas de ellas lo hicieron de manera alarmante. Sólo Benito Juárez y Miguel Hidalgo 


\section{Cuadro 1}

\section{Índice de desarrollo humano}

\begin{tabular}{lccc}
\hline Componentes del IDH & 2000 & 2004 & Variación Porcentual \\
\hline Salud & & & \\
Nacional & 0.8171 & 0.825 & $0.97 \%$ \\
DF & 0.8346 & 0.8401 & $0.66 \%$ \\
Educación & & & \\
Nacional & 0.8163 & 0.8331 & $2.06 \%$ \\
DF & 0.8945 & 0.9031 & $0.96 \%$ \\
Ingreso & & & \\
Nacional & 0.7485 & 0.7513 & $0.37 \%$ \\
DF & 0.9127 & 0.9079 & $-0.53 \%$ \\
Total & & & \\
Nacional & 0.794 & 0.8031 & $1.15 \%$ \\
DF & 0.8806 & 0.8837 & $0.35 \%$ \\
\hline
\end{tabular}

Fuente: United Nations Development Programme, Human Development Report-Mexico 2006-2007. Migration and Human Development, 2007.

mantuvieron su clasificación -primero y cuarto lugar a nivel nacional, respectivamente- y sólo Cuajimalpa mejoro su posición.

El cuadro 2 muestra el valor del IDH y la clasificación a nivel nacional en los años 2000 y 2005 para las 16 delegaciones de la ciudad de México, también muestra las variaciones en la clasificación y las variaciones porcentuales en el periodo; se observa que Milpa Alta, la demarcación con el IDH más bajo en la ciudad de México, perdió 343 posiciones al pasar del lugar 284 al lugar 627 de un total de 2,454 municipios. Magdalena Contreras, Xochimilco e Iztapalapa, que están dentro de las cinco delegaciones con menor IDH perdieron más de 100 posiciones. El cuadro 2 también muestra que, entre 2000 y 2005, el incremento promedio del IDH en toda la ciudad fue de $3.00 \%$, el incremento promedio en las cinco delegaciones con mayor IDH fue de $4.19 \%$, es decir, mayor al promedio total de la ciudad, mientras que el crecimiento promedio en las cinco delegaciones con menor IDH fue de tan sólo 1.79, por debajo del promedio total en la ciudad de México. Estas cifras demuestran cómo se acentúa la polarización y la desigualdad social y económica en esta demarcación, tomando en cuenta que el IDH se integra por tres componentes: salud, educación e ingreso.

El cuadro 3 muestra el IDH de cada delegación y el país con el índice más aproximado a cada una de ellas para el año 2005, también señala la posición de cada país a nivel internacional. Las cifras internacionales se obtienen de Human Development Report 2007/2008, (Undp, 2007a). En el cuadro se aprecia que la delegación puntera: Benito Juárez tiene un índice similar al de Estados Unidos o España, y estos países ocupan la 


\section{Cuadro 2}

\section{Índice de desarrollo humano en las delegaciones del Distrito Federal y su posición a nivel nacional}

\begin{tabular}{|c|c|c|c|c|c|c|}
\hline \multirow[b]{2}{*}{ Delegación } & 2000 & Clasificación & \multicolumn{2}{|r|}{2005} & \multicolumn{2}{|c|}{ Diferencias 05 vs 00} \\
\hline & $I D H$ & nacional & $I D H$ & $\begin{array}{c}\text { Clasificación } \\
\text { Nacional }\end{array}$ & Clasificación & $\%$ \\
\hline Benito Juárez & 0.9164 & 1 & 0.9509 & 1 & 0 & 3.76 \\
\hline Miguel Hidalgo & 0.8816 & 4 & 0.9188 & 4 & 0 & 4.22 \\
\hline Coyoacán & 0.8837 & 3 & 0.9169 & 5 & -2 & 3.76 \\
\hline $\begin{array}{l}\text { Cuajimalpa } \\
\text { de Morelos }\end{array}$ & 0.8426 & 35 & 0.8994 & 15 & 20 & 6.74 \\
\hline Cuauhtémoc & 0.8699 & 5 & 0.8921 & 23 & -18 & 2.55 \\
\hline Azcapotzalco & 0.8551 & 14 & 0.8915 & 25 & -11 & 4.26 \\
\hline Tlalpan & 0.8616 & 10 & 0.8791 & 53 & -43 & 2.03 \\
\hline Iztacalco & 0.8503 & 22 & 0.8765 & 60 & -38 & 3.08 \\
\hline Venustiano Carranza & 0.8498 & 23 & 0.8740 & 74 & -51 & 2.85 \\
\hline Álvaro Obregón & 0.8536 & 18 & 0.8719 & 81 & -63 & 2.14 \\
\hline Gustavo A. Madero & 0.8420 & 37 & 0.8700 & 85 & -48 & 3.33 \\
\hline $\begin{array}{l}\text { La Magdalena } \\
\text { Contreras }\end{array}$ & 0.8445 & 30 & 0.8558 & 136 & -106 & 1.34 \\
\hline Xochimilco & 0.8348 & 56 & 0.8481 & 172 & -116 & 1.59 \\
\hline Tláhuac & 0.8212 & 102 & 0.8473 & 177 & -75 & 3.18 \\
\hline Iztapalapa & 0.8284 & 75 & 0.8463 & 183 & -108 & 2.16 \\
\hline Milpa Alta & 0.7930 & 284 & 0.7983 & 627 & -343 & 0.67 \\
\hline Promedios & & & & & & \\
\hline Promedio 5 mayores & 0.8788 & & 0.9156 & & & 4.19 \\
\hline Promedio 5 menores & 0.8244 & & 0.8392 & & & 1.79 \\
\hline Promedio Total & 0.8518 & & 0.8773 & & & 3.00 \\
\hline
\end{tabular}

Fuente: Índice de Desarrollo Humano municipal en México 2000-2005, UNDP (2008) y cálculos propios.

posición 12 y 13 a nivel internacional. Por otra parte, la delegación con el mayor rezago en cuanto a desarrollo humano se refiere: Milpa Alta, tiene un índice similar al de Dominica, isla-estado localizada en el mar Caribe, y este país ocupa la posición 71 a nivel internacional. Estas cifras muestran cómo dentro de la entidad federativa que ocupa el territorio más pequeño de todas las entidades del país existe un marcado y creciente contraste en cuanto a desarrollo humano se refiere.

Entre el 2002 y el 2006 los ingresos del gobierno de la ciudad de México se incrementaron, aunque en buena medida este aumento fue por mayores aportaciones y participaciones de la federación y por incremento de la deuda. Los rubros de gastos que se vieron más beneficiados, entre 2000 y 2006, con el aumento de los ingresos, fueron el de subsidios, transferencias y ayudas, que prácticamente se triplicó, y el de obras públi- 


\section{Cuadro 3}

\section{Comparativo de las delegaciones a nivel internacional (2005)}

\begin{tabular}{|c|c|c|c|c|}
\hline Delegación & $I D H$ & $\begin{array}{l}\text { ifica } \\
D F\end{array}$ & País con IDH similar & $\begin{array}{c}\text { Posición del } \\
\text { pais a nivel } \\
\text { mundial }\end{array}$ \\
\hline Benito Juárez & 0.9509 & 1 & $\begin{array}{l}\text { Estados Unidos, } \\
\text { Espańa }\end{array}$ & 12,13 \\
\hline Miguel Hidalgo & 0.9188 & 2 & Korea & 26 \\
\hline Coyoacán & 0.9169 & 3 & Slovenia & 27 \\
\hline Cuajimalpa de Morelos & 0.8994 & 4 & Portugal & 29 \\
\hline Cuauhtémoc & 0.8921 & 5 & $\begin{array}{l}\text { Barbados, Republica } \\
\text { Checa }\end{array}$ & 31,32 \\
\hline Azcapotzalco & 0.8915 & 6 & Kuwait & 33 \\
\hline Tlalpan & 0.8791 & 7 & Malta & 34 \\
\hline Iztacalco & 0.8765 & 8 & Qatar & 35 \\
\hline Venustiano Carranza & 0.8740 & 9 & Hungría & 36 \\
\hline Álvaro Obregón & 0.8719 & 10 & Polonia & 37 \\
\hline Gustavo A. Madero & 0.8700 & 11 & Argentia & 38 \\
\hline La Magdalena Contreras & 0.8558 & 12 & Latvia & 45 \\
\hline Xochimilco & 0.8481 & 13 & Croacia & 47 \\
\hline Tláhuac & 0.8473 & 14 & Costa Rica & 48 \\
\hline Iztapalapa & 0.8463 & 15 & Bahamas & 49 \\
\hline Milpa Alta & 0.7983 & 16 & Dominica & 71 \\
\hline
\end{tabular}

Fuente: Índice de Desarrollo Humano Municipal en México 2000-2005, undp (2008) y Human Development Report, UndP, 2007.

cas y acciones sociales el cual registró un incremento de $72 \%$ en el periodo (INEGI, 2008). Aunque estos dos conceptos de gasto tienen una orientación social y de crecimiento económico, no es muy claro que tan efectivo y eficiente haya sido su ejercicio en el periodo analizado, principalmente cuando las cifras de ingreso promedio, crecimiento económico e índice de desarrollo humano muestran crecimientos lentos y menores al de la media nacional.

\section{Comparaciones internacionales}

El análisis anterior es consistente con la teoría neoclásica, que indica que las regiones o países con mayor ingreso promedio tienden a tener menor crecimiento, es decir, el ingreso promedio inicial es inversamente proporcional al crecimiento económico (Galor, 1996; Sala-i-Martin, 1996; Thirlwall, 2002: 20-28). Si el concepto de capital lo entendemos de una manera amplia, de tal forma que involucre capital físico y capital humano, entonces la teoría neoclásica está también en posibilidades de explicar 
el lento crecimiento del IDH en el Distrito Federal, como se comentó previamente. En esta sección se analiza si la expansión de indicadores en dicha circunscripción es semejante a comparaciones internacionales o si existe margen para que, en la ciudad, haya un mayor crecimiento de indicadores económicos y de desarrollo humano.

El cuadro 4 muestra el ingreso per cápita de dos bloques de países (ingreso alto e ingreso medio alto) y de dos regiones en México (Distrito Federal y estados), a precios de 2,000 para los años 2000 y 2005 . La clasificación de países por nivel de ingreso se hace de acuerdo al Banco Mundial (2007), y sus promedios y variaciones se obtienen mediante cálculos propios con cifras de la base estadística World Development Indicators, del Banco Mundial (2007a). Las cifras de ingreso per cápita de las entidades federativas se obtienen mediante cálculos propios con cifras del PIB del INEGI (2008) y con cifras de población de Conapo (2008a). Para efectos comparativos, los dos bloques de países que se utilizan, son debido a que la ciudad de México entra en el rango de ingreso alto, mientras que el ingreso promedio del resto de los estados entra en el rango de ingreso medio alto.

\section{Cuadro 4 \\ Ingreso per cápita promedio por bloques de países y por regiones en México (2000 USD)}

\begin{tabular}{lrrc}
\hline \multicolumn{1}{c}{ Ingresos per cápita } & 2000 & 2005 & Crecimiento anual \\
\hline Países de ingreso alto & 22,836 & 25,173 & $2.05 \%$ \\
Países de ingreso medio alto & 5,068 & 5,809 & $2.92 \%$ \\
DF & 15,041 & 15,182 & $0.19 \%$ \\
Estados sin DF & 5,060 & 5,359 & $1.18 \%$ \\
\multicolumn{4}{c}{ Variaciones de crecimiento anual del ingreso } \\
\multicolumn{2}{c}{ Puntos porcentuales } \\
Alto $v$ medio alto & -0.88 & \\
DF $v$ s estados sin DF & -0.99 & \\
\hline
\end{tabular}

Fuente: Cálculos propios con cifras de Banco Mundial, World Development Indicators, CD-Rom, (2007a). Instituto Nacional de Estadística Geografía e Informática, Banco de Información Económica, 2008. Consejo Nacional de Población, Indicadores demográficos básicos 1990-2030, (2008a).

En el cuadro 4 se observa que entre más alto es el ingreso promedio en los bloques de países menor es su crecimiento anual promedio, de igual forma se demuestra que el crecimiento del ingreso en la ciudad de México es menor que en el resto de los estados, lo cual es consistente con la teoría neoclásica. Las celdas inferiores del cuadro 4 muestran datos interesantes. El crecimiento anual del ingreso per cápita en países de ingreso alto es menor en 0.88 puntos porcentuales con respecto a los países con ingreso medio alto, mientras que el ingreso per cápita en la capital crece 
0.99 puntos porcentuales menos que el resto de las entidades federativas. En general, la expansión del ingreso per cápita ha sido lenta en todo el país, sin embargo, estas cifras sugieren que la ciudad de México tiene posibilidades de crecer más y cumplir con su papel de polo catalizador de la economía nacional.

Las cifras son más reveladoras cuando se utilizan datos del pIB. El cuadro 5 muestra que entre 2000 y 2005 el crecimiento promedio anual del pIB en los países de ingreso alto fue de $2.18 \%$ y en los países de ingreso medio alto fue de $2.87 \%$, es decir, una diferencia de 0.69 puntos porcentuales. En el mismo periodo, el crecimiento promedio anual del PIB en el Distrito Federal fue de $0.05 \%$ y en el resto de los estados fue de $2.38 \%$, lo cual arroga una diferencia de 2.33 puntos porcentuales. En este sentido, las entidades federativas sin el Distrito Federal crecen de manera similar a los estándares internacionales y de acuerdo a su nivel de ingreso, pero el Distrito Federal crece a un ritmo muy lento en relación a su nivel de ingreso; es decir, el crecimiento de la capital retarda el crecimiento nacional, considerando que el Distrito Federal aún ostenta alrededor del $20 \%$ del PIB nacional.

\section{Cuadro 5 \\ Crecimiento anual del PIB 2000-2005 \\ por bloques de países y por regiones en México (2000 USD)}

\begin{tabular}{lc}
\hline \multicolumn{1}{c}{$P I B$} & $\begin{array}{c}\text { Crecimiento anual } \\
2000-2005\end{array}$ \\
\hline Países de ingreso alto & $2.18 \%$ \\
Países de ingreso medio alto & $2.87 \%$ \\
DF & $0.05 \%$ \\
Estados sin DF & $2.38 \%$ \\
Variaciones de crecimiento anual del PIB & \\
Puntos porcentuales & \\
Alto vs medio alto & -0.69 \\
DF $v$ estados sin DF & -2.33 \\
\hline
\end{tabular}

Fuente: Cálculos propios con cifras de Banco Mundial, World Development Indicators, CD-Rom, 2007. Instituto Nacional de Estadística Geografía e Informática, Banco de Información Económica, 2008.

Por último, el cuadro 6 muestra un comparativo de crecimiento del IDH. El periodo para los bloques de países es 2000-2005 (UNDP 2007a), y el periodo para las entidades federativas en México es 2000-2004 (UNDP 2007). Las cifras obtenidas de este cuadro revelan tendencias que concuerdan con las presentadas previamente.

La primera sección del cuadro 6 indica que los países con ingreso alto aumentan su IDH en $0.29 \%$ promedio anual, mientras que los países con ingreso medio alto lo hacen en $0.46 \%$, lo cual es también consistente con 


\begin{tabular}{|c|c|c|c|}
\hline \multicolumn{4}{|c|}{$\begin{array}{c}\text { Cuadro } 6 \\
\text { Crecimiento del IDH por bloques de países y } \\
\text { por regiones en México }\end{array}$} \\
\hline & 2000 & 2005 & $\begin{array}{c}\text { Crecimiento } \\
\text { anual }\end{array}$ \\
\hline Países de ingreso alto & 0.931 & 0.945 & $0.29 \%$ \\
\hline Países de ingreso medio alto & 0.828 & 0.847 & $0.46 \%$ \\
\hline \multicolumn{3}{|c|}{ Variación de crecimiento en puntos porcentuales } & -0.17 \\
\hline & 2000 & 2004 & $\begin{array}{c}\text { Crecimiento en } \\
\text { el periodo }\end{array}$ \\
\hline $\mathrm{DF}$ & 0.881 & 0.884 & $0.09 \%$ \\
\hline Estados sin DF & 0.784 & 0.795 & $0.34 \%$ \\
\hline \multicolumn{3}{|c|}{ Variación de crecimiento en puntos porcentuales } & -0.25 \\
\hline
\end{tabular}

Fuente: Cálculos propios con cifras de United Nations Development Programme, Human Development Report-2007/2008, Fighting Climate Change: Human Solidarity in a Divided World, 2007a. United Nations Development Programme, Human Development Report-Mexico 2006-2007 - Migration and Human Development, 2007.

los supuestos de la teoría neoclásica. La diferencia en el crecimiento del IDH entre los dos bloques de países es de 0.17 puntos porcentuales. En lo que respecta a regiones en México, el Distrito Federal crece $0.09 \%$ promedio anual y el resto de los estados lo hace en $0.34 \%$, lo cual arroga una diferencia de 0.25 puntos porcentuales, mayor a la presentada entre bloques de países. En general, el IDH en México crece de manera más lenta que las tendencias internacionales, pero las entidades federativas $\sin$ la ciudad de México se apegan más a la tendencia de los países con ingreso medio alto, mientras que el crecimiento del IDH en la capital está muy por debajo de la tendencia de países con ingreso alto. Esto indica que la ciudad de México tiene potencial para incrementar con mayor velocidad su IDH, comparado con estándares internacionales, y que su actual ritmo de crecimiento retarda el incremento del IDH a nivel nacional.

\section{Análisis paramétrico de convergencia y causalidad}

En esta sección se realizan tres procedimientos econométricos: el primero es un análisis de datos de panel, se emplea el software estadístico Limdep en el que se utilizan observaciones de corte transversal de cada una de las 32 entidades federativas, a través de 13 observaciones anuales entre el periodo 1994-2006. En este primer caso se evalúa si el PIB per cápita de las entidades federativas de menor ingreso converge con el de las de mayor ingreso. La primera ecuación a estimar es la siguiente: 


$$
P I B P c_{i t}=\alpha+\beta(P I B P c I N I)_{i t}+\varepsilon_{i t}
$$

Donde PIBpc representa el PIB per cápita, PIBpcINI es el PIB per cápita inicial, los subíndices $i, t$ representan entidad federativa y año respectivamente, el término de error $\varepsilon$ se asume que satisface supuestos de ruido blanco, $\alpha$ es un intercepto constante y $\beta$ es el parámetro por obtener. La ecuación (1) se estima con cifras absolutas y en logaritmos.

Si el signo de $\beta$ es negativo y estadísticamente significativo, entonces el modelo representaría una situación en la que los estados de menor ingreso crecen más rápido que los de mayor ingreso, indicando convergencia de tipo incondicional, dado que en el modelo no se permiten variaciones en alguna otra característica económica, social o política de las entidades al no incluir variables adicionales de control. Es decir, la ecuación 1 sería una representación de la teoría neoclásica, donde se asume que el nivel o crecimiento del PIB per cápita depende exclusivamente del PIB per cápita inicial, mientras que el resto de las variables que afectan al crecimiento son iguales a través de las regiones o países en estudio.

Los resultados para las ecuaciones con cifras absolutas y en logaritmos se presentan en las columnas uno y dos, respectivamente, del cuadro 7. En ambas ecuaciones el signo de $\beta$ es positivo y estadísticamente significativo, indicando divergencia entre entidades. Ambos modelos se estiman con el método de Mínimos Cuadrados Ordinarios (MCO), no se utiliza la especificación de Efectos Fijos porque la variable exógena, PIB per cápita inicial, no varía a través del tiempo y no es posible obtener su coeficiente. ${ }^{1}$ Los resultados del modelo de Efectos Aleatorios no se reportan porque son muy similares a los obtenidos mediante MCo.

Para confirmar la divergencia entre entidades, en la columna tres se presentan los resultados de evaluar la relación entre el nivel del PIB per cápita (en cifras absolutas) y el crecimiento del mismo (en logaritmos), mediante la aplicación de la siguiente ecuación:

$$
\operatorname{LnPIB} p c_{i t}=\alpha+\beta(\operatorname{PIB} p c)_{i t}+\varepsilon_{i t}
$$

La especificación que se reporta es MCo para hacerla comparable con los ejercicios anteriores.

${ }^{1}$ El modelo de Efectos Fijos, también conocido como modelo de Efectos Dentro de Grupo, tiene la siguiente especificación:

$$
y_{i t}-\overline{y_{i}}=\left(X_{i t}-\overline{X_{i}}\right) \beta+\left(u_{i t}-\overline{u_{i}}\right)
$$

Donde la barra representa el promedio de cada grupo; luego entonces, si todas las observaciones dentro de un grupo a través del tiempo son iguales, no existe variabilidad dentro de grupos y no es posible estimar el parámetro $\beta$. 
En este caso $\beta$ resultó nuevamente positivo y significativo, lo que indica que las entidades con mayor nivel de PIB per cápita tienden a crecer más rápido que las que tienen un nivel más bajo en esta variable. ${ }^{2}$ Aunque esta tendencia no necesariamente aplica para el caso del Distrito Federal, dado la evidencia estadística que se presentó en las secciones anteriores.

Antes de establecer alguna conclusión es necesario realizar un ejercicio adicional abandonando los supuestos neoclásicos. Para tal fin tomamos los postulados de la Nueva Teoría del Crecimiento, la cual establecen que el nivel o crecimiento de PІв per cápita depende de diversos factores endógenos, principalmente de la inversión en capital humano y capital físico que impulse la innovación y la productividad del trabajo (Lucas, 1988; Rebelo, 1991; Romer, 1994). En este sentido agregamos a la ecuación 1 las variables de población $(P O B)$ que representa capital humano, esperanza de vida (ESPVID) que representa nivel de desarrollo humano e inversión extranjera directa per cápita (IEDPC) que es una proxy de capital físico, para obtener la siguiente:

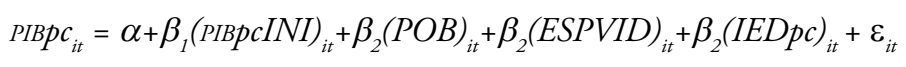

Los resultados, utilizando valores absolutos y logaritmos se reportan en las columnas cuatro y cinco, respectivamente, del cuadro 7.

Una vez que se incorporan las variables de control, y con esto se permiten variaciones económicas y sociales a través de las entidades federativas, $\beta$ permanece positiva y estadísticamente significativa. La variable de población es positiva y estadísticamente significativa cuando la ecuación se presenta en logaritmos, esto sugiere que las entidades que más crecen en términos de población tienden a presentar mayores niveles de crecimiento económico; por el contrario, ésta no es significativa cuando las cifras se encuentran en valores absolutos, y por lo tanto no hay evidencia para decir que existe una relación entre nivel de PIB per cápita y tamaño de población. Los coeficientes de las variables de esperanza de vida e IED también presentan signos positivos y son estadísticamente significativos, tanto con cifras absolutas como en logaritmos. Es decir, las entidades federativas con mayores niveles de PIB per cápita tienden a acumular capital físico y desarrollo humano con mayor velocidad y a crecer más rápido a través de un proceso endógeno, lo cual propicia la divergencia entre regiones.

2 También se estimaron los modelos de Efectos Fijos y Efectos Aleatorios, controlando en ambos casos efectos de grupo y efectos de grupo y período, en todas las especificaciones se obtiene una $\beta$ positiva, estadísticamente significativa y con magnitudes similares, lo cual es un indicativo de la robustez de los resultados. 


\section{Cuadro 7 \\ Divergencia entre entidades federativas}

\begin{tabular}{|c|c|c|c|c|c|}
\hline & \multicolumn{2}{|c|}{$\begin{array}{l}\text { Convergencia } \\
\text { incondicional }\end{array}$} & \multirow[b]{2}{*}{ 3) LnPIвpc } & \multicolumn{2}{|c|}{$\begin{array}{c}\text { Convergencia } \\
\text { condicional }\end{array}$} \\
\hline & 1) $P I B p c$ & 2) $L n P I B p c$ & & 4) $P I B p c$ & 5) $\operatorname{LnPIBpc}$ \\
\hline PIBpcINI & $\begin{array}{r}0.868 \\
(0.000)\end{array}$ & & & $\begin{array}{r}0.630 \\
(0.000)\end{array}$ & \\
\hline LnPIBpcINI & & $\begin{array}{r}0.949 \\
(0.000)\end{array}$ & & & $\begin{array}{r}0.813 \\
(0.000)\end{array}$ \\
\hline PIBpc & & & $\begin{array}{r}6.450 \mathrm{E}-05 \\
(0.000)\end{array}$ & & \\
\hline POB & & & & $\begin{array}{r}1.016 \mathrm{E}-05 \\
(0.824)\end{array}$ & \\
\hline LnPOB & & & & & $\begin{array}{r}0.044 \\
(0.000)\end{array}$ \\
\hline ESPVID & & & & $\begin{array}{r}1,234.153 \\
(0.000)\end{array}$ & \\
\hline LnESPVID & & & & & $\begin{array}{r}6.337 \\
(0.000)\end{array}$ \\
\hline IEDpc & & & & $\begin{array}{r}8.160 \\
(0.000)\end{array}$ & \\
\hline LnIEDpc & & & & & $\begin{array}{r}0.012 \\
(0.000)\end{array}$ \\
\hline
\end{tabular}

Fuente: Cálculos propios con cifras de INEGI (2008) para el caso de PIB e Inversión Extranjera Directa, Conapo (2008a) para el caso de esperanza de vida y población. Las cifras per cápita se calculan con los datos de población de Conapo (2008a). Valores $p$ en paréntesis.

Este primer análisis paramétrico sugiere que las entidades más desarrolladas en México (incluido el Distrito Federal) no operan como polos catalizadores o diseminadores, porque no hay evidencia de convergencia entre regiones.

El segundo análisis econométrico es también un estudio de datos de panel, que evalúa la convergencia del ingreso per cápita entre municipios, en este caso se utiliza la información de UNDP (2008), que contiene cifras a nivel municipal para los años 2000 y 2005 . En total se emplean 2,418 datos de corte transversal para los dos periodos mencionados, lo que arroga un total de 4,836 observaciones. La ecuación que se estima para probar convergencia es la siguiente:

$$
I P C_{i t}=\alpha+\beta\left(\text { IPCini }_{i t}+\varepsilon_{i t}\right.
$$

Donde IPC e IPCini son el ingreso per cápita e ingreso per cápita inicial, respectivamente, los subíndices $i, t$ representan municipio y año 
en el mismo orden, y los signos $\varepsilon, \alpha$ y $\beta$ corresponden a los conceptos mencionados en el ejercicio anterior. Los resultados obtenidos de la ecuación 4, tanto en cifras absolutas como en logaritmos, se muestran en las columnas uno y dos del cuadro 8, en ellas se aprecia que no existe un proceso de convergencia incondicional de tipo neoclásico. Los resultados de la ecuación 5, presentados en la columna tres, confirman que los municipios con mayor ingreso promedio tienden a crecer más rápido que los que tienen ingresos menores.

$$
\operatorname{LnIPC}_{i t}=\alpha+\beta(I P C)_{i t}+\varepsilon_{i t}
$$

A través de la ecuación 6 se prueba si existe un proceso de convergencia condicional de tipo endógeno, los resultados se muestran en las columnas cuatro y cinco del cuadro 8 , tanto en valores absolutos como en logaritmos. Nuevamente se aprecia una tendencia de divergencia en los ingresos a nivel nacional, en este caso entre municipios. Las variables de control utilizadas son población $(P O B)$, tasa de mortalidad infantil (TASMOI) $\mathrm{y}$ tasa de asistencia escolar (TASASE).

$$
I P C_{i t}=\alpha+\beta_{1}(\text { IPCini })_{i t}+\beta_{2}(P O B)_{i t}+\beta_{2}(\text { TASMOI })_{i t}+\beta_{2}(\text { TASASE })_{i t}+\varepsilon_{i t}
$$

Los resultados presentados en el cuadro 8 fueron obtenidos empleando el método de MCO, con base en los argumentos explicados en el primer análisis paramétrico.

El tercer análisis econométrico es un estudio de series de tiempo, el cual se realiza con 67 observaciones anuales entre 1940 y 2006 para dos series, el pib de la ciudad de México y el del resto de las entidades. En este caso se evalúa la causalidad del crecimiento económico entre ambas series, es decir, se busca probar si la ciudad de México afecta el crecimiento del resto del país o viceversa. La muestra se divide en tres subperiodos, prácticamente con el mismo número de observaciones cada uno (1940-1961, 1962-1983 y 1984-2006), con el fin de analizar si la causalidad cambia a lo largo del tiempo, la ecuación que se estima es la siguiente:

$$
\ln P I B s n D F_{t}-\ln P I B s n D F_{t-1}=\beta\left(\ln P I B D F_{t-1}-\ln P I B D F_{t-2}\right)+\varepsilon_{t}-\varepsilon_{t-1}
$$

La cual se puede abreviar de la siguiente forma:

$$
\Delta \ln P I B s n D F_{t}=\beta\left(\Delta \ln P I B D F_{t-1}\right)+\Delta \varepsilon_{t}
$$

Donde PIBSADF representa el PIB de las entidades federativas sin incluir la ciudad de México, PIBDF representa el PIB de la ciudad de México, el 


\section{Cuadro 8 \\ Divergencia entre municipios}

\begin{tabular}{|c|c|c|c|c|c|}
\hline & \multicolumn{2}{|c|}{$\begin{array}{l}\text { Convergencia } \\
\text { incondicional }\end{array}$} & \multirow[b]{2}{*}{ 3) $\operatorname{LnIPC}$} & \multicolumn{2}{|c|}{$\begin{array}{c}\text { Convergencia } \\
\text { condicional }\end{array}$} \\
\hline & 1) IPC & 2) $\operatorname{LnIPC}$ & & 4) $I P C$ & 5) $\mathrm{LnIPC}$ \\
\hline \multirow[t]{2}{*}{ IPCini } & 0.938 & & & 0.768 & \\
\hline & $(0.000)$ & & & $(0.000)$ & \\
\hline \multirow[t]{2}{*}{ LnIPCini } & & 0.819 & & & 0.632 \\
\hline & & $(0.000)$ & & & $(0.000)$ \\
\hline \multirow[t]{2}{*}{ IPC } & & & $1.667 \mathrm{E}-04$ & & \\
\hline & & & $(0.000)$ & & \\
\hline \multirow[t]{2}{*}{ POB } & & & & 0.001 & \\
\hline & & & & $(0.000)$ & \\
\hline \multirow[t]{2}{*}{ LnPOB } & & & & & 0.006 \\
\hline & & & & & $(0.012)$ \\
\hline \multirow[t]{2}{*}{ TASMOI } & & & & -84.404 & \\
\hline & & & & $(0.000)$ & \\
\hline \multirow[t]{2}{*}{ LnTASMOI } & & & & & -0.497 \\
\hline & & & & & $(0.024)$ \\
\hline \multirow[t]{2}{*}{ TASASE } & & & & 10.190 & \\
\hline & & & & $(0.001)$ & \\
\hline \multirow[t]{2}{*}{ LnTASASE } & & & & & 0,076 \\
\hline & & & & & $(0.000)$ \\
\hline
\end{tabular}

Fuente: Cálculos propios con cifras de UNDP (2008). Valores p en paréntesis.

subíndice $t$ indica los años, el término de error $\varepsilon$ se asume que satisface supuestos de ruido blanco, y $\beta$ es el parámetro por encontrar. La ecuación 7 se estima en logaritmos para obtener tasas de crecimiento y se expresa en primeras diferencias (la diferencia se representa con el símbolo $\Delta$ ) para eliminar problemas de autocorrelación y obtener estimaciones cointegradas. Adicionalmente, el término del lado derecho de la ecuación (PIBDF) se rezaga un periodo para poder captar sus efectos en la variable endógena. Los resultados se presentan en el cuadro 9.

La regresión correspondiente al periodo 1940-1961 muestra un coeficiente $\beta$ positivo y estadísticamente significativo, indicando de esta forma una asociación entre el crecimiento del рів de la ciudad de México y el crecimiento nacional durante este lapso de tiempo. La prueba de causalidad de Granger muestra que los efectos son bidireccionales, es decir, el PIB de la ciudad de México causa el crecimiento de las demás entidades federativas y viceversa.

Para el periodo 1962-1983 el coeficiente también es positivo y estadísticamente significativo. Sin embargo, la prueba de causalidad de Granger indica que la direccionalidad del efecto sólo es en un sentido, el 
Cuadro 9

Efecto del PIB del DF en las entidades federativas y análisis de causalidad

\begin{tabular}{lccc}
\hline & \multicolumn{3}{c}{ Periodos } \\
\hline & $1940-1961$ & $1962-1983$ & $1984-2006$ \\
PIBDF & 0.653 & 1.040 & -0.265 \\
& $(0.000)$ & $(0.000)$ & $(0.238)$ \\
Estadístico Durbin-Watson & 1.876 & 1,524 & 2.044 \\
Prueba ADF & $(0.002)$ & $(0.002)$ & $(0.000)$ \\
Constante & $(0.009)$ & $(0.000)$ & $(0.000)$ \\
Constante y tendencia & $(0.000)$ & $(0.041)$ & $(0.000)$ \\
Ninguno & & & \\
Prueba de causalidad de Granger & & & \\
PIBDF no causa pib sin DF & $(0.047)$ & $(0.133)$ & $(0.504)$ \\
PIB sin df no causa PIBDF & $(0.000)$ & $(0.000)$ & $(0.000)$ \\
Observaciones & 22 & 22 & 23 \\
\hline
\end{tabular}

Fuente: Cálculos propios con cifras de INEGI (2008).

Valores $p$ en paréntesis. Variable dependiente es el PIB nacional sin el DF.

PIв de la capital nacional no causa el PIB de las demás entidades federativas pero si hay efecto de manera inversa.

En el tercer periodo 1984-2006, el coeficiente $\beta$ pierde su nivel de significancia, y la prueba de Granger confirma que el pIB de la ciudad de México no causa el pIB de las demás entidades federativas.

Los valores del estadístico Durbin-Watson sugieren que no hay evidencia de autocorrelación en las tres regresiones. Adicionalmente, la prueba Dicky-Fuller, bajo tres especificaciones, revela la no existencia de raíz unitaria en el término de error, en otras palabras, las series de cada ecuación están cointegradas y por lo tanto, el tipo de relación que pueda existir en cada regresión es de largo plazo.

Los resultados ilustran que en una etapa inicial, previa a los años sesenta, la ciudad de México influía en el crecimiento nacional; posteriormente el efecto se perdió en dicha dirección y en décadas recientes no existe una clara evidencia de que el crecimiento de dicha demarcación y el resto del país estén relacionados.

\section{Conclusiones}

Este estudio muestra que la ciudad de México ostenta los indicadores de PIB, PIB per cápita y desarrollo humano más altos a nivel nacional, pero a su vez, estos indicadores tienen crecimientos menores que el resto de las 
entidades federativas en México. Esta tendencia está en concordancia con la teoría neoclásica del crecimiento y, por consiguiente, podría esperarse un proceso de convergencia a nivel nacional.

Sin embargo, el estudio revela, a través de comparaciones internacionales, que el Distrito Federal tiene potencial para crecer más aceleradamente en términos económicos y de desarrollo humano a pesar de tener altos indicadores en términos relativos. Adicionalmente se muestra, con un análisis paramétrico, que no existe un proceso de convergencia a nivel nacional. Por el contrario, existe un proceso de divergencia incondicional y condicional, en el que las entidades y municipios con mayor nivel de ingreso crecen más rápido que los de menor pIв per cápita, pero la ciudad de México no se ajusta a este proceso, debido a su lento crecimiento.

Por otra parte, a través del análisis paramétrico de causalidad desarrollado en este artículo, se aprecia que en una etapa inicial (entre 1940 y 1961), la ciudad de México ejercía un efecto positivo en el crecimiento nacional, es decir, cumplía con un papel de polo catalizador. En los periodos subsecuentes este efecto se pierde, y en el último periodo analizado (1984-2006) no sólo la ciudad de México no causa el crecimiento a nivel nacional, sino que tampoco hay evidencia de una relación significativa entre el crecimiento de la capital y las demás entidades. En otras palabras, la ciudad de México ha perdido su papel de polo catalizador a nivel nacional y no ejerce un efecto diseminador de tipo dualista.

Con base en el análisis previo, se sugieren las siguientes acciones en términos de política pública:

Debe existir una política regional y de desconcentración más activa, en la que se asigne mayor gasto en educación, salud, infraestructura y creación de empleos en las regiones menos desarrolladas, con el fin de que estas crezcan más aceleradamente y se propicie un proceso de convergencia.

Por otra parte, es importante crear cadenas productivas y vínculos económicos y sociales entre las entidades con mayores niveles de desarrollo e ingreso y el resto de los estados. En el caso del Distrito Federal, por ejemplo, el equipo de transporte público (vagones de metro, autobuses articulados, autos eléctricos) podría adquirirse en el interior del país para impulsar la producción y el desarrollo tecnológico en otras regiones. De igual forma, la ciudad de México podría aprovechar su potencial en términos de capital humano calificado, y la planta universitaria con la que cuenta, para impulsar programas de desarrollo tecnológico. Por último, es necesario replantear en la ciudad de México el gasto social y la inversión pública y privada, de tal forma que se retome el crecimiento económico acorde al potencial de la entidad y, de esta manera, esté en posibilidades de ejercer su papel de polo catalizador. 
Con estas acciones es posible estimular el mercado interno, desarrollar tecnología, substituir importaciones y generar el proceso de diseminación requerido para la convergencia y el desarrollo equilibrado entre entidades.

\section{Bibliografía}

BM (Banco Mundial) (2007), "Clasificación de países por ingreso, country classification", Washington DC, http://web.worldbank.org/ WBSITE/EXTERNAL/DATASTATISTICS, septiembre de 2008.

вм (Banco Mundial) (2007a), World development indicators, CD-Rom, Washington DC.

Barro, Robert J. y Xavier Sala-i-Martin (2003), Economic Growth, MIT Press, Boston, pp. 23-84.

Conapo (Consejo Nacional de Población) (2008), "Proyecciones de la población de México 2005-2050", http://www.conapo.gob. $\mathrm{mx} / 00$ cifras/5.htm, agosto de 2008.

Conapo (Consejo Nacional de Población) (2008a), "Indicadores demográficos básicos 1990-2030”, http://www.conapo.gob.mx/00cifras/ 00indicadores.htm, agosto de 2008.

Galor, Oded (1996), "Convergence? Inferences from theoretical models", The Economic Journal, 106 (437), Blackwell Publishers, Oxford, pp. 1056-1080.

German-Soto, Vicente (2005), "Generación del Producto Interno Bruto mexicano por entidad federativa 1940-1992", El Trimestre Económico, 72 (3), Fondo de Cultura Económica, México, pp. 617-653.

Hirschman, Albert O. (1958), Strategy of economic development, Yale University Press, New Haven.

INEGI (Instituto Nacional de Estadística Geografía e Informática) (2008), "Banco de Información Económica", http://dgcnesyp.inegi.org. $\mathrm{mx} /$ cgi-win/bdieintsi.exe, julio de 2008.

Lucas, Robert E. (1988), "On the mechanics of economic development", Journal of Monetary Economics, 22, University of Chicago, Chicago, pp. 3-42. 
Myrdal, Gunnar (1957), Economic theory and underdeveloped regions, Duckworth, London.

Presidencia de la República (2006), Sexto Informe de Gobierno del Presidente de los Estados Unidos Mexicanos, ciudad de México, http://sexto.informe.fox.presidencia.gob.mx/index.php? idseccion $=4$, septiembre de 2008 .

Ranis, Gustav (2003), "Is dualism worth revisiting?”, University of Yale Economic Growth Center Discussion Paper 870, http://ssrn.com/ abstract $=464240$, febrero de 2012 .

Rebelo, Sergio (1991), "Long-run policy analysis and the long-run growth", Journal of Political Economy, 99 (3), National Bureau of Economic Research, Cambridge, pp. 500-521.

Romer, Paul M. (1994), “The origins of endogenous growth”, The Journal of Economic Perspectives, 8 (1), American Economic Association, Pittsburgh, pp. 3-22.

Sala-i-Martin, Xavier (1996), "The classical approach to convergence analysis”, Economic Journal, 106 (437), Royal Economic Society, Oxford, pp. 1019-1036.

Saldaña-Zorrilla, Sergio O. (2008), "Dualismo y polarización histórica en Iberoamérica”, Revista El Cotidiano, 149, Universidad Autónoma Metropolitana-Azcapotzalco, México, pp. 104-114.

SEP (Secretaría de Educación Pública) (2008), Estadísticas históricas por estado, http://www.sep.gob.mx/wb/sep1/sep1_Estadisticas, julio de 2008.

Solow, Robert (1956), "A contribution to the theory of economic growth", Quarterly Journal of Economics, 70 (1), Oxford University Press, Oxford, pp. 65-94.

Swan, Trevor (1956), "Economic growth and capital accumulation", Economic Record, 32, Wiley, Melbourne, pp. 334-361.

Thirlwall, Anthony P. (2002), The nature of economic growth, an alternative framework for understanding the performance of nations, Edward Elgar, Cheltenham. 
Thirlwall, Anthony P. (2006), Growth and development: with special reference to developing countries, Palgrave Macmillan, New York.

UNDP (United Nations Development Programme) (2007), Human development report-Mexico 2006-2007-Migration and human development, Programas Educativos, México.

undP (United Nations Development Programme) (2007a), Human development report-2007-2008, Fighting climate change: human solidarity in a divided world, Palgrave Macmillan, New York.

UNDP (United Nations Development Programme) (2008), Índice de desarrollo humano municipal en México 2000-2005, Producción Creativa, México.

Recibido: 6 de noviembre de 2008. Reenviado: 24 de enero de 2012. Aceptado: 13 de diciembre de 2012.

Gerardo Ángeles-Castro. Es doctor en economía por la Universidad de Kent, Inglaterra. Actualmente es profesor-investigador en la Escuela Superior de Economía y coordinador de la red de desarrollo económico del Instituto Politécnico Nacional. Es miembro del Sistema Nacional de Investigadores, nivel I. Sus líneas de investigación son crecimiento y desarrollo económico y desigualdad de ingresos. Entre sus últimas publicaciones destacan: "The effects of trade and foreign direct investment on inequality: do governance and macroeconomic stability matters?", Economía Mexicana. Nueva época, xx (1), México, pp. 181-215, (2011); "Economic liberalization and income distribution: theory and evidence in México" en Ángeles-Castro, Perrotini-Hernández y Ríos-Bolívar, Growth and economic development in Latin America, ediciones market liberalism, Routledge, uK, pp. 195-219, (2011); en coautoría, "Desregulación económica y flexibilidad laboral: una forma de reducir el costo laboral", Economía y Sociedad, 27 (xvir), Universidad Michoacana de San Nicolás de Hidalgo, Morelia, pp. 65-83, (2011); en coautoría, Valuación de opciones sobre indices bursátiles y determinación de la estructura de plazos de las tasas de interés en un modelo de equilibrio general, Investigación Económica, Conapo (271), México, pp. 43-80, (2010). 